\title{
A cruise ship emergency medical evacuation triggered by handheld ultrasound findings and directed by tele-ultrasound
}

\author{
Keith S. Boniface ${ }^{1}$, Neal Sikka ${ }^{1}$, Nicholas Page, Asi Peretz, Hamid Shokoohi ${ }^{1,2}{ }^{\circledR}$ \\ ${ }^{1}$ The George Washington University, Washington, DC, United States \\ ${ }^{2}$ Massachusetts General Hospital, Boston, MA, United States
}

\begin{abstract}
Cruise ships travel far from shoreside medical care and present a unique austere medical environment. For the cruise ship physician, decisions regarding emergency medical evacuation can be challenging. In the event that a passenger or crew member becomes seriously ill or is injured, the use of point-of-care ultrasound may assist in clarifying the diagnosis and stratifying the risk of a delayed care, and at times expedite an emergent medical evacuation. In this report we present the first case reported in the literature of an emergency medical evacuation from a cruise ship triggered by handheld ultrasound. A point-of-care ultrasound performed by a trained cruise ship physician, reviewed by a remote telemedical consultant with experience in point-of-care ultrasound, identified an ectopic pregnancy with intraabdominal free fluid in a young female patient with abdominal pain and expedited emergent helicopter evacuation from a cruise ship to a shoreside facility, where she immediately underwent successful surgery. The case highlights a medical evacuation that was accurately triggered by utilising a handheld ultrasound and successfully directed via a tele-ultrasound consultation. American College of Emergency Physicians (ACEP) health care guidelines for cruise ship medical facilities should be updated to include guidelines for point-of-care ultrasound, including training and telemedical support.
\end{abstract}

(Int Marit Health 2020; 71, 1: 42-45)

Key words: cruise ship medical evacuation, point-of-care ultrasound, ectopic pregnancy, tele-ultrasound

\section{INTRODUCTION}

The cruise industry estimates that 28.5 million passengers took part in a cruise in 2018 - an increase of $7 \%$ from the previous year - for an average of 7 days [1]. This number can only be expected to grow, as over 120 new ships are on order at shipyards around the world with an average capacity of over 2000 passengers, as well as crew members [2]. It is inevitable that some passengers and crew members will become seriously ill or injured while at sea, at times far away from shoreside medical facilities. Medical care aboard cruise ships is guided by American College of Emergency Physicians (ACEP) Section of Cruise Medicine's Guidelines [3]. The guideline makes recommendations for training of physicians and nurses, medications, and equipment on board to ensure that passengers and crew who become ill or injured while at sea receive the best care possible in the austere maritime environment. On board, patients are cared for by the physicians and nurses of the ship's medical centre, and the vast majority of these patients are managed on board until resolution or until the next scheduled port call. Rarely, when patient's needs exceed onboard capabilities, a diversion or emergent medical evacuation is required. Decision on an emergency medical evacuation is one of the most complex decisions the cruise ship physician has to make. Often, these decisions are undertaken with incomplete data due to the limitations of diagnostic testing on board. Increasing diagnostic certainty can help the physician make the decision to divert a ship or evacuate a patient with more accuracy and greater confidence. 
In recent years, point-of-care ultrasound (POCUS) performed by clinicians at the bedside has transformed diagnostics in emergency medicine [4]. At George Washington University, we operate a not-for-profit academic maritime medical advisory service. Our Maritime Medical Access telemedicine group, along with ultrasound faculty, have provided assistance to a cruise line through the acquisition of ultrasound equipment, development of POCUS protocols, training of physicians and nurses in POCUS, and tele-ultrasound support and interpretation after deployment of ultrasound equipment.

We present a case where ultrasound led to a dramatic shift in a patient's care and prompted helicopter evacuation. This case highlights a successful medical evacuation that was accurately triggered by utilising a handheld ultrasound and successfully directed via a tele-ultrasound practice.

\section{CASE REPORT}

A 25-year-old female crewmember presented to the ship's medical centre at 16:35 hours on a sea complaining of abdominal pain for 1 day. The pain had been of varying intensity but was now 10 out of 10 in severity. Her pain was more prominent in the right lower quadrant. There was no reported vaginal bleeding or discharge. Vital signs were normal, and the patient was afebrile. Examination was significant for a soft abdomen, with positive tenderness and voluntary guarding of the right lower quadrant. Absent were rebound tenderness or signs of an acute abdomen. The remainder of her examination was normal. Pelvic examination was deferred.

Laboratory evaluation was significant for a white blood cell count of $14 \times 10^{3}$ per mcl, a haematocrit of $29.7 \%$, platelets of $247 \times 10^{3}$ per mcl, a C-reactive protein level of $<5 \mathrm{mg} / \mathrm{L}$, and normal renal function and electroIytes. A urine pregnancy test was performed that indicated a new diagnosis of pregnancy; a urinalysis was normal. Last menstrual period dating placed the pregnancy at 8 weeks 3 days gestation.

Considering a wide range of important differential diagnoses including ectopic pregnancy, ovarian torsion, ruptured ovarian cyst, and appendicitis, a transabdominal pelvic ultrasound was performed by the cruise ship physician utilising a handheld ultrasound. With the finding suggestive of a ruptured ectopic pregnancy, the shoreside telemedicine team was consulted and images were transferred for review by the shoreside telemedicine team including an emergency physician with tele-ultrasound experience. The ultrasound revealed a uterus empty of a gestational sac, or any signs of intrauterine pregnancy including a yolk sac or foetal pole (Fig. 1). In addition, the ultrasound demonstrated the presence of a complex cystic mass in the adnexa (Fig. 2), and free intraperitoneal fluid in the right upper quadrant (Fig. 3).

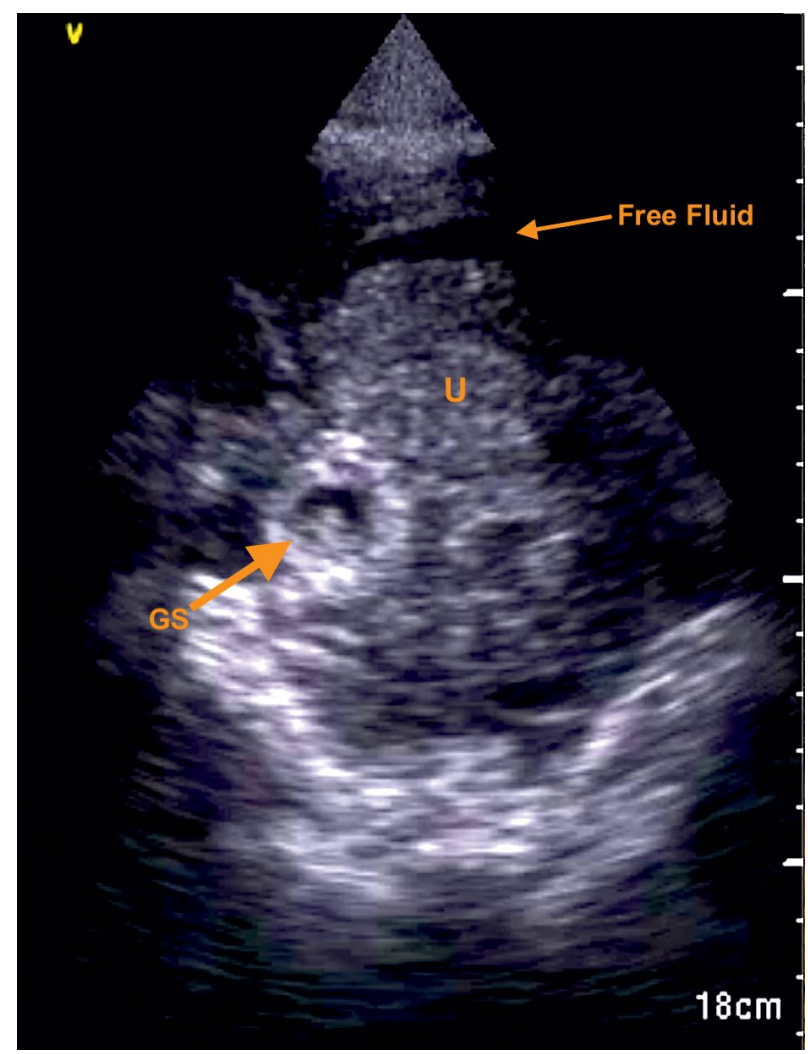

Figure 1. Transabdominal pelvic ultrasound image from a 23-year-old 8-week pregnant crewmember. Transverse view shows empty uterus $(U)$ surrounded by anechoic free fluid (arrow), with visualised gestational sac (GS) and foetal pole to the right adnexa encircled by hyperechoic decidual ring

The vessel was at sea, with the next port call scheduled for the following morning at 08:00. As the next port had no surgical facilities, the decision was made to coordinate a helicopter evacuation with the United States Coast Guard. The evacuation was initiated by 18:31. At the point of evacuation, she had become tachycardic and hypotensive, consistent with a clinical presentation of early haemorrhagic shock. After coordination with the receiving institution, she was flown directly to a shoreside hospital, where the diagnosis of ruptured ectopic pregnancy and haemoperitoneum was confirmed. She was transferred to the operating room and underwent an emergent laparoscopic salpingectomy.

\section{DISCUSSION}

This is the first case that we have identified in the medical or maritime literature where ultrasound performed by the onboard cruise ship staff led to a successful emergency evacuation of a patient with life threatening clinical condition, resulting in expeditious surgical management. Cruise lines almost without exception prohibit pregnant women who will be in their $24^{\text {th }}$ week of gestation at any point during the cruise, limiting potential medical complications 


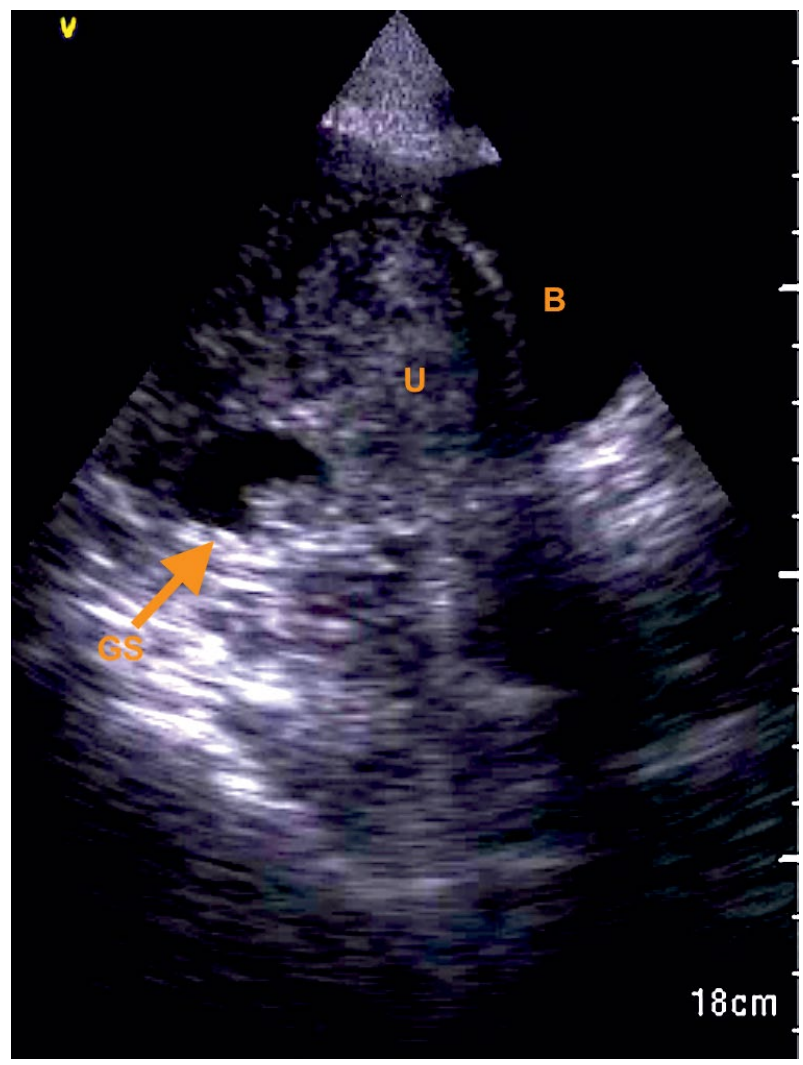

Figure 2. Transabdominal pelvic ultrasound image from a 23-year-old 8-week pregnant crewmember. Sagittal view shows empty uterus (U) with gestational sac (GS) and foetal pole can be seen in adjacent right adnexa. A thin rim of free fluid is noted around the fundus of the uterus; $B$ - bladder

for patients with viable pregnancies [5]. Ectopic pregnancies often present during first and early second trimester, however, and therefore are to be found in the cruising population of pregnant women. A high index of suspicion for ruptured ectopic pregnancy is required, and ultrasound findings can help to increase clinical suspicion of ruptured ectopic pregnancy (free peritoneal fluid, empty intrauterine cavity, adnexal mass) as well as haemodynamic instability because of the resultant haemoperitoneum. Presence of free fluid in Morison's pouch may predict the need for operative management [6]. Ruptured ectopic pregnancy combines two limitations of care found in the maritime medicine environment - absent surgical capabilities, and lack of blood products.

Point-of-care ultrasound is a skill that can be very helpful to the cruise medicine physician, and a review of the ACEP' emergency ultrasound guidelines point to several indications useful to physicians working in cruise medicine who face cases of shock, vaginal bleeding, dyspnoea, abdominal pain, chest pain, change in vision, and swollen extremities, all syndromes where a diagnosis can be made easily with POCUS [4].

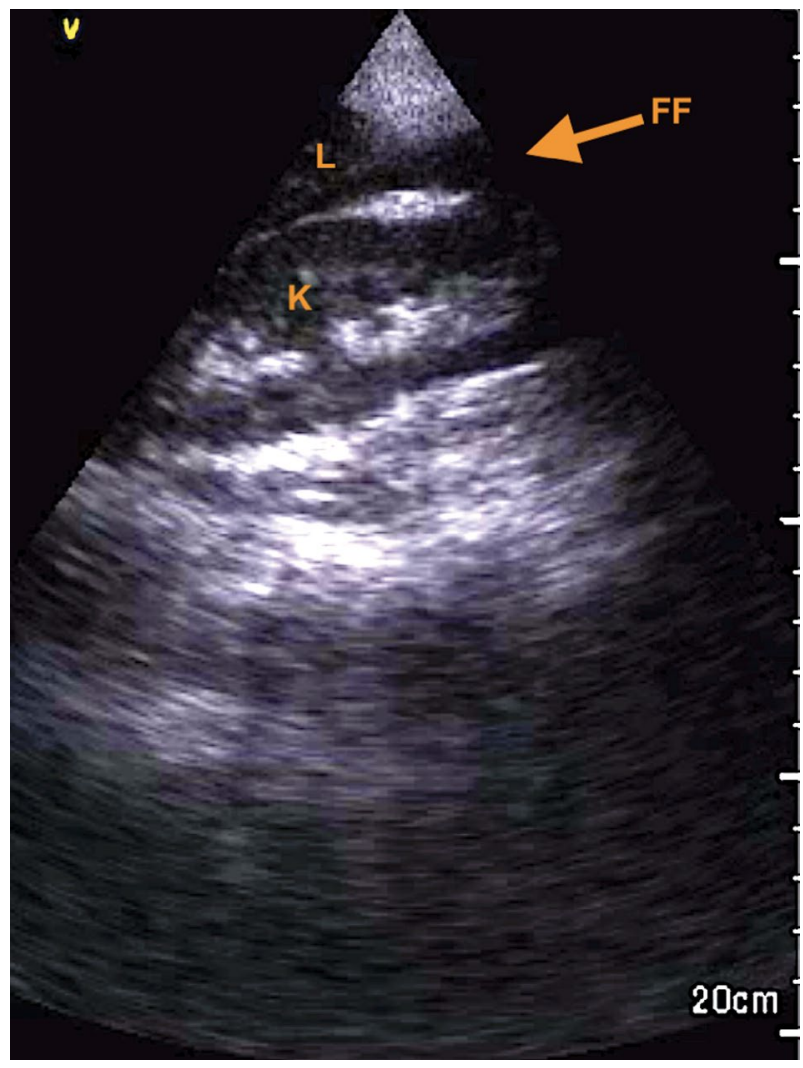

Figure 3. Transabdominal pelvic ultrasound image from a 23-year-old 8-week pregnant crewmember. Right upper quadrant view shows free intraperitoneal fluid (FF) in Morison's pouch; $L$ - liver; $\mathrm{K}$ - right kidney

Ultrasound equipment has evolved rapidly over the past two decades, with large radiology cart-based systems being replaced at the point of care by smaller cart-based machines, laptop-sized machines, and truly handheld ultrasounds that can connect to a tablet or smart phone. Factors that should influence decisions about which machine to buy include what type of examinations will be done, whether superficial (e.g. for vascular access and foreign body imaging) and deep structures (e.g. transabdominal obstetrical, cardiac, and abdominal imaging) will both be imaged (as this requires two probes for vast majority of manufacturers), cost, plan for storage of images, and image quality.

Protocols for ultrasound imaging should be focused on clinically important diagnoses that impact the course of treatment for patients aboard ships. Identification of surgical emergencies such as ruptured ectopic pregnancy and intraperitoneal bleeding is paramount, as it can take critical time to get a patient at sea transferred to the care of a surgeon. Lung and cardiac ultrasound can expedite the diagnosis of patients with congestive heart failure, pneumonia, right heart strain, and pericardial effusion, leading to earlier administration of the correct therapy for patients with 
unclear causes of hypotension or dyspnoea. Normal findings can be reassuring or help to rule out certain diagnoses.

One of the limiting factors for implementation of POCUS aboard cruise ships is the need for initial and ongoing training of onboard physicians. Cruise ships move 7 days a week, 52 weeks a year, providing little time to set aside for initial training or refresher courses. New physicians frequently rotate to the ship's medical centre, creating an ongoing need for initial training. Our team has found that structuring training into a port call at the home port at the end of one cruise and before the beginning of the next is feasible. One team of trainers can train multiple medical centre teams over several days, as other ships come to the home port. Pre-recording lectures to maximize hands-on scanning time during these trainings makes the best use of the small training window.

Tele-ultrasound is the use of technology to bring ultrasound from the bedside of a patient to the screen of a remote expert [7]. We use a store-and-forward tele-ultrasound system with a commercially available cloud service. Ultrasound images are obtained by the ship's physicians at the bedside and sent to our telemedicine and tele-ultrasound team along with clinical information and initial image interpretation. Our tele-ultrasound specialists may request further views to ensure that the patients receive the appropriate diagnosis and targeted therapy for their condition.

We suggest that future revisions of the ACEP Health Care Guidelines for Cruise Ship Medical Facilities should include POCUS, and cruise lines should ensure that physicians working on board have both access to ultrasound equipment as well as to training and ongoing quality assurance.

\section{CONCLUSIONS}

This case report highlights an emergent medical evacuation in a case with ruptured ectopic pregnancy that was initiated by utilising a handheld ultrasound, successfully directed via tele-ultrasound consultation. The ultrasound findings consistent with a ruptured ectopic pregnancy led to a helicopter evacuation to a coastal hospital, where the patient received expedited definitive surgical management. Training cruise ship physicians to perform POCUS and establishing tele-ultrasound and maritime protocols may streamline clinical decision-making. Future revisions of the ACEP Health Care Guidelines for Cruise Ship Medical Facilities should address the use of POCUS, and cruise lines should ensure that physicians working on cruises board have access to ultrasound equipment as well as to training, tele-medical support, and ongoing quality assurance.

\section{REFERENCES}

1. Cruise Lines International Association (CLIA) reveals growth in global and North American passenger numbers and insights. Retrieved from. https://cruising.org/news-and-research/press-room/2019/ april/clia-reveals-growth (Accessed August 18, 2019).

2. Cruise Industry News, "Cruise Ship Orderbook". Retrieved from. https://www.cruiseindustrynews.com/pdf (Accessed August 18, 201).

3. Health care guidelines for cruise ship medical facilities. Policy resource and education paper. January 2019. Retrieved from. https://www.acep.org/globalassets/new-pdfs/preps/health-careguidelines-for-cruise-ship-medical-facilities--prep.pdf (Accessed December 6, 2019).

4. Ultrasound Guidelines: Emergency, Point-of-Care and Clinical Ultrasound Guidelines in Medicine. Ann Emerg Med. 2017; 69(5): e27-e54, doi: 10.1016/j.annemergmed.2016.08.457, indexed in Pubmed: 28442101.

5. Cruise Compete. Pregnancy policies by cruise line. Retrieved from. https://www.cruisecompete.com/pressrelease010810. php (Accessed August 18, 2019).

6. Moore C, Todd WM, O'Brien E, et al. Free fluid in Morison's pouch on bedside ultrasound predicts need for operative intervention in suspected ectopic pregnancy. Acad Emerg Med. 2007; 14(8): 755-758, doi: 10.1197/j.aem.2007.04.010, indexed in Pubmed: 17554008.

7. Britton N, Miller MA, Safadi S, et al. Tele-Ultrasound in resource-limited settings: a systematic review. Front Public Health. 2019; 7: 244, doi: 10.3389/fpubh.2019.00244, indexed in Pubmed: 31552212. 